\title{
The Accuracy of Initial Bone Cutting in Total Knee Arthroplasty
}

\author{
Takaaki Ohmori1, Toru Maeda1,2, Tamon Kabata1* ${ }^{*}$, Yoshitomo Kajino', \\ Shintaro Iwai ${ }^{1}$, Hiroyuki Tsuchiya ${ }^{1}$ \\ ${ }^{1}$ Department of Orthopedic Surgery, Graduate School of Medical Science, Kanazawa University, Kanazawa, \\ Japan \\ ${ }^{2}$ Department of Orthopedic Surgery, Kanazawa Red Cross Hospital, Kanazawa, Japan \\ Email: ${ }^{*}$ tamonkabata@yahoo.co.jp
}

Received 28 August 2015; accepted 27 September 2015; published 30 September 2015

Copyright (C) 2015 by authors and Scientific Research Publishing Inc.

This work is licensed under the Creative Commons Attribution International License (CC BY).

http://creativecommons.org/licenses/by/4.0/

(c) (i) Open Access

\section{Abstract}

Background: The aim of this study was to evaluate the accuracy of initial bone cutting of the distal femur and the proximal tibia in TKA using an image-free navigation system. Methods: From February 2006 to March 2013, we evaluated 60 knees in 50 patients using an image-free navigation system (Navigation: Stryker Navigation Cart System; Software: Stryker Knee Navigation; Ver2.0: Stryker Orthopaedics US NJ Mahwah). First, we measured the angle shown by the navigation system before cutting, at the time we set the jig. Second, we measured the angles shown by navigation after the bone was cut using the jig. Then, we compared these two angles for each patient to determine the bone cutting error. Results: In the distal femur, 37 of 60 knees were cut in an extended position in the sagittal plane, and 26 of 60 knees were cut in a varus in the coronal plane. In the proximal tibia, 29 of 60 knees were cut with decreased posterior slope in the sagittal plane, and 26 of 60 knees were cut in a valgus. Conclusions: In this study, the distal femur tended to be cut in an extended and a varus position and the proximal tibia did with decreased posterior slope and in a valgus position after initial bone cutting. It is necessary to note the initial cutting error in TKA. Since cutting errors affect postoperative outcome, we should cut bones several times. And as the reasons of the cause of the error, we propose new reason that cutting bone is not parallel with accuracy to AP axis.

\section{Keywords}

Total Knee Arthroplasty, Bone Cutting Errors, Distal Femur, Proximal Tibia, Navigation

\footnotetext{
${ }^{*}$ Corresponding author.
}

How to cite this paper: Ohmori, T., Maeda, T., Kabata, T., Kajino, Y., Iwai, S. and Tsuchiya, H. (2015) The Accuracy of Initial Bone Cutting in Total Knee Arthroplasty. Open Journal of Orthopedics, 5, 297-304. 


\section{Introduction}

Worldwide, many total knee arthroplasty (TKA) operations are performed each year. Many factors affect clinical outcomes. Appropriate alignment in sagittal and coronal plane is one of the most important among them, and component installation is one of the most important factors of appropriate alignment. In the sagittal plane, malalignment can affect size of components and postoperative range of motion of the knee [1] [2]. And in the coronal plane, it has been reported that a cutting error of more than $3^{\circ}$ worsens the prognosis of TKA [3]-[5]. There have been few studies about the accuracy of bone cutting in TKA, because it is heavily dependent on the skill of the surgeon [6]. Nakahara et al. examined the angular difference between the cutting surface and the preoperatively planned angle in the distal femur using a CT based navigation system [6]. However, in that study it was possible that cutting errors were caused not only by inaccurate bone cutting but also by inaccurate reproduction of the preoperative plan. The aim of our study was to evaluate the accuracy of initial bone cutting of the distal femur and the proximal tibia in TKA using an image-free navigation system with more precision than previous studies. The goal was to objectively evaluate only the effect of bone cutting.

\section{Materials and Methods}

From February 2006 to March 2013, we performed 390 TKA and UKA in our hospital. In this study, we investigated only primary TKA by same team. Exclusion criteria included UKA, revision TKA and tumor knee prosthesis. 29 UKA, 92 revision TKA and 30 tumor knee prosthesis were excluded. Moreover 125 were also excluded because not performed by our team. Out of 114 primary TKA, 54 had incomplete data. The analysis was conducted on 60 primary TKA implanted in 50 patients ( 8 men and 42 women).The average patient's age was 72.5 years (range 50 - 89 years). The diagnoses for all patients were 42 knee osteoarthritis, 7 rheumatoid arthritis and 1 osteonecrosis (Figure 1). We used an image-free navigation system (Navigation: Stryker Navigation Cart System, Software: Stryker Knee Navigation Ver2.0: Stryker Orthopaedics US NJ Mahwah) in all cases at the time of the surgery. The saw blade was $1.27 \mathrm{~mm}$ thick and $18 \mathrm{~mm}$ wide, and the slot thickness of the cutting block was $1.40 \mathrm{~mm}$.

As an anatomical index for the image-free navigation system, we set the mechanical axes of both the femur and tibia by the following method. First, in the femur, we registered at the center of femoral head, the lateral and medial epicondyles of femur, the Whiteside line, and the knee center. Next, in the tibia, we registered at the anterior-posterior axis, lateral and medial malleolus of ankle, and the knee center. On the femur side, we installed a jig perpendicularly to a mechanical axis in a coronal plane. In a sagittal plane, we installed the jig at about $2^{\circ}$ -

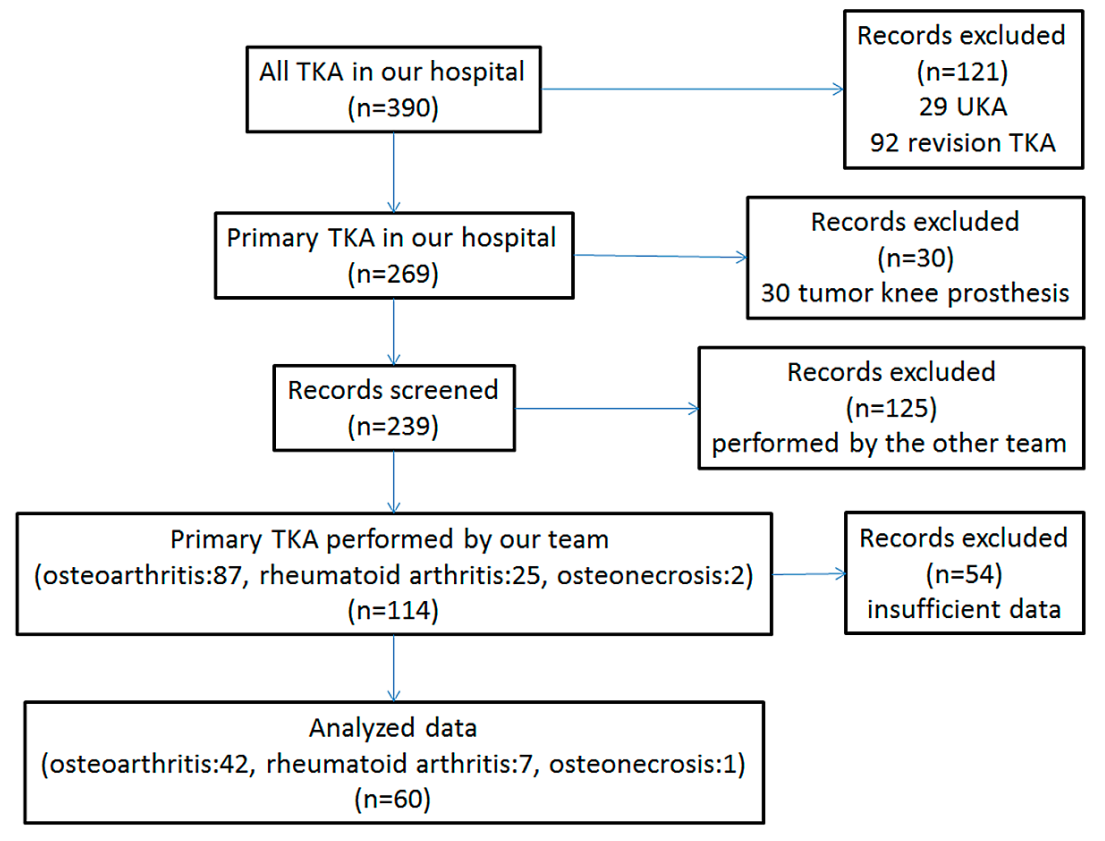

Figure 1. Process of selection of this study. 
$3^{\circ}$ flexed position from the mechanical axis.

For evaluation, we defined + or - as the difference of direction shown by the navigation system. First, we measured the angle shown by the navigation system before cutting, at the time we set the jig. Second, we measured the angles shown by navigation after the bone was cut using the jig. Then, we subtracted the angle shown by the navigation system before cutting from the angle after cutting by the navigation system to determine the bone cutting error.

We evaluated the coronal and sagittal planes on both femur and tibia. In the coronal plane on the femur, we regarded an error of the valgus position as a + and of the varus position as a -; in the sagittal plane, an error of the extended position was regarded as + and of the flexed position as - (Figure 2(a)). On the tibia, we measured

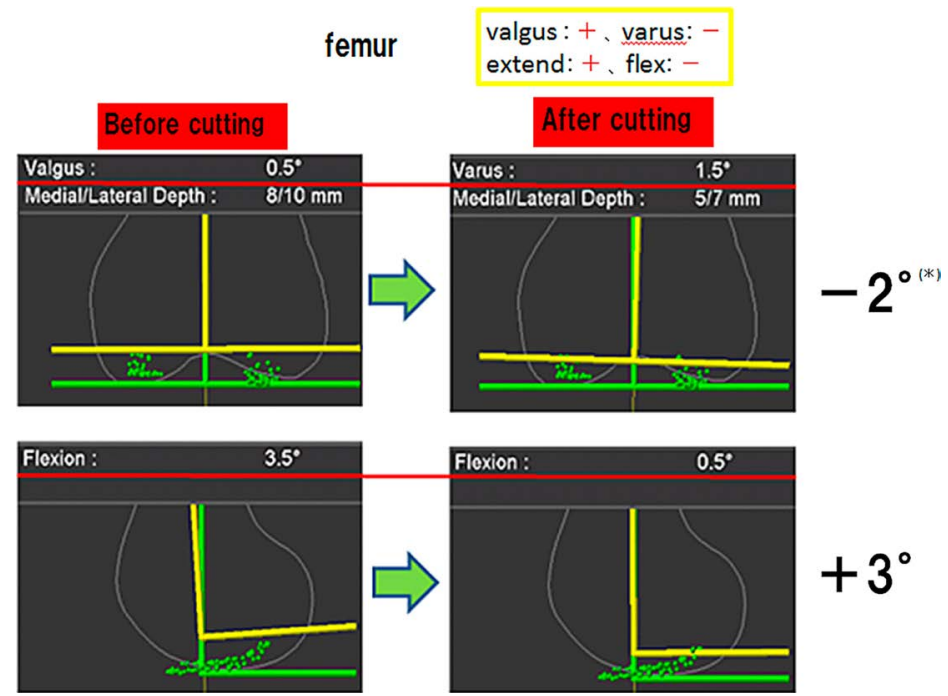

( ) Since the angle shown by the navigation system was valgus $0.5^{\circ}$ before cutting, we defined it as $+0.5^{\circ}$. Since the angle shown by the navigation system was varus $1.5^{\circ}$ after cutting, we defined it as $-1.5^{\circ}$. Then we subtracted $+0.5^{\circ}$ from $-1.5^{\circ}$. In the result, cutting error was $-2.0^{\circ}$ (varus $2.0^{\circ}$ ).

(a)

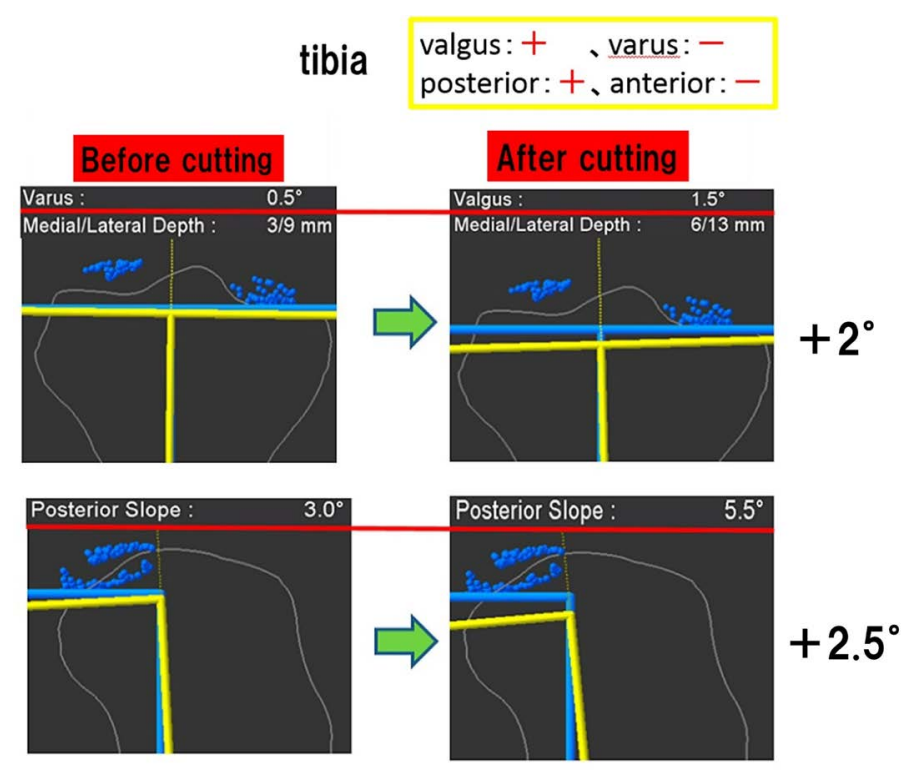

(b)

Figure 2. Method of error evaluation on the femur and tibia. (a) On the femur Error of valgus position as + and varus position as - in the coronal plane (upper side). Error of extended position as + and flexed position as - in the sagittal plane (lower side). (b) On the tibia Error of valgus position as + and varus position as - in the coronal plane (upper side). Error of posterior position as + and anterior position as - in the sagittal plane (lower side). 
similarly in the coronal plane; in the sagittal plane, we regarded an error of the posterior position as + and anterior position as - (Figure 2(b)). The Chi-square test was used to consider the difference of how to cut in each situation.

The same team performed all operations. All surgical procedures were performed by a senior surgeon and 2 3 assistants. All measurements were performed by one investigator who did not participate to the surgical procedure. An inter observer error was not occurred, because they just tried to record the value of the screen. The Chi-square test was used to consider the difference of how to cut in each situation.

All procedures were approved by the Institutional Review Board of Kanazawa University.

The patients provided informed consent for the publication of this report.

\section{Results}

On the distal femur side, 37 (62\%) of 60 knees were cut in an extended position, and $14(23 \%)$ were cut in a flexed position in the sagittal plane (Figure 3(a)) $(\mathrm{P}<0.01)$. The average absolute error was $1.4^{\circ}$. Of the 60 knees,

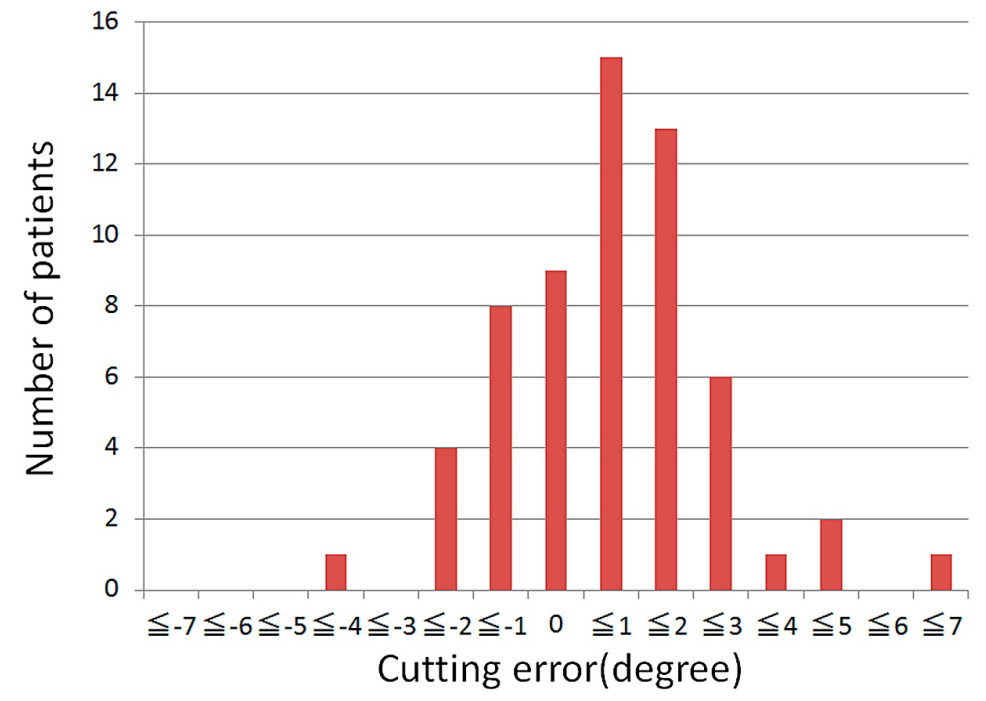

(a)

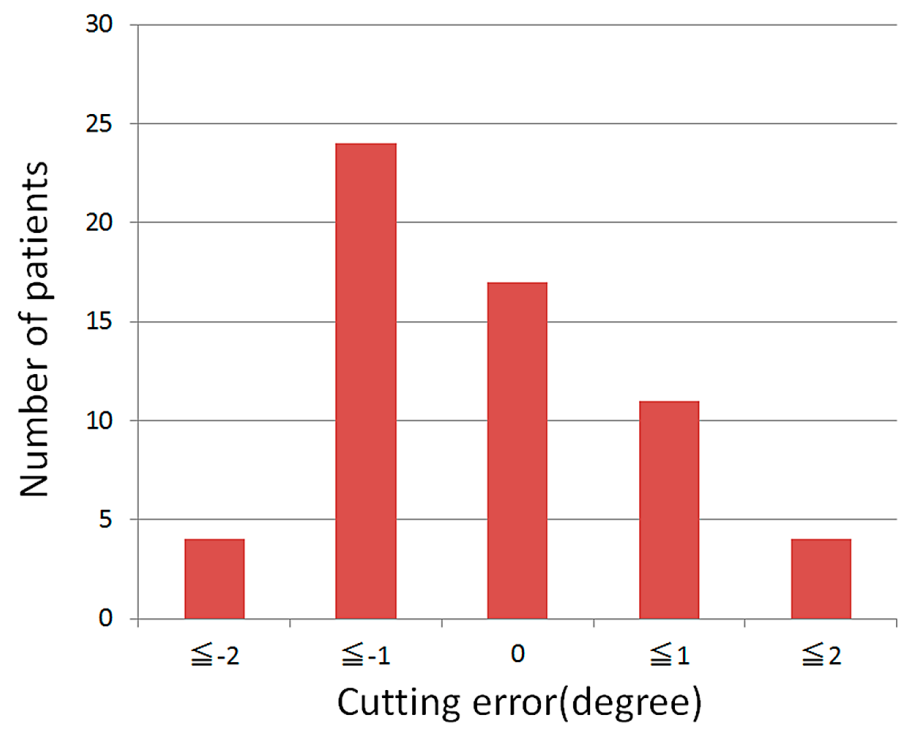

(b)

Figure 3. Dot line indicate border of each direction Distal femur cutting error. (a) In the sagittal plane. A positive value indicates extended position. (b) In the coronal plane. A positive value indicates valgus position. 
28 had error more than $1^{\circ}$. In the coronal plane, $26(43 \%)$ of 60 knees were cut on a varus, and 17 of 60 knees were cut on a valgus (Figure 3(b)) $(\mathrm{P}>0.05)$. The average absolute error was $0.64^{\circ}$. Eight $(13 \%)$ of 60 knees had error more than $1^{\circ}$. There was no knee with an average absolute error value of more than $2^{\circ}$.

On the proximal tibia side, 29 (48\%) of 60 knees were cut with a decreased posterior slope, and $18(30 \%)$ were cut with an increased posterior slope in the sagittal plane (Figure 4(a)) $(\mathrm{P}>0.05)$. The average absolute error was $1.10^{\circ}$. There were $24(40 \%)$ of 60 knees with average absolute error values of more than $1^{\circ}$. In the coronal plane, $21(35 \%)$ of 60 knees were cut on a varus, and $26(48 \%)$ were cut on a valgus (Figure 4(b)) (P > $0.05)$. Seven (12\%) of the 60 knees had average absolute error values of more than $1^{\circ}$. The average absolute error was $0.60^{\circ}$. No knee registered an average absolute error of more than $2^{\circ}$.

We evaluated the bone cutting relationship between the femur and the tibia in a coronal plane. Results showed that $7(12 \%)$ of 60 knees were cut on a valgus on both the femur and the tibia side, and $8(13 \%)$ were cut on a varus on both the femur and the tibia side. A total of 15 knees were cut in the same direction. On the other hand, in $7(12 \%)$ of 60 knees the femur were cut on a valgus and the tibia was cut on a varus, and in $10(17 \%)$ the femur was cut on a varus and the tibia were cut on a valgus. Altogether 17 knees were cut in different directions (Table 1).

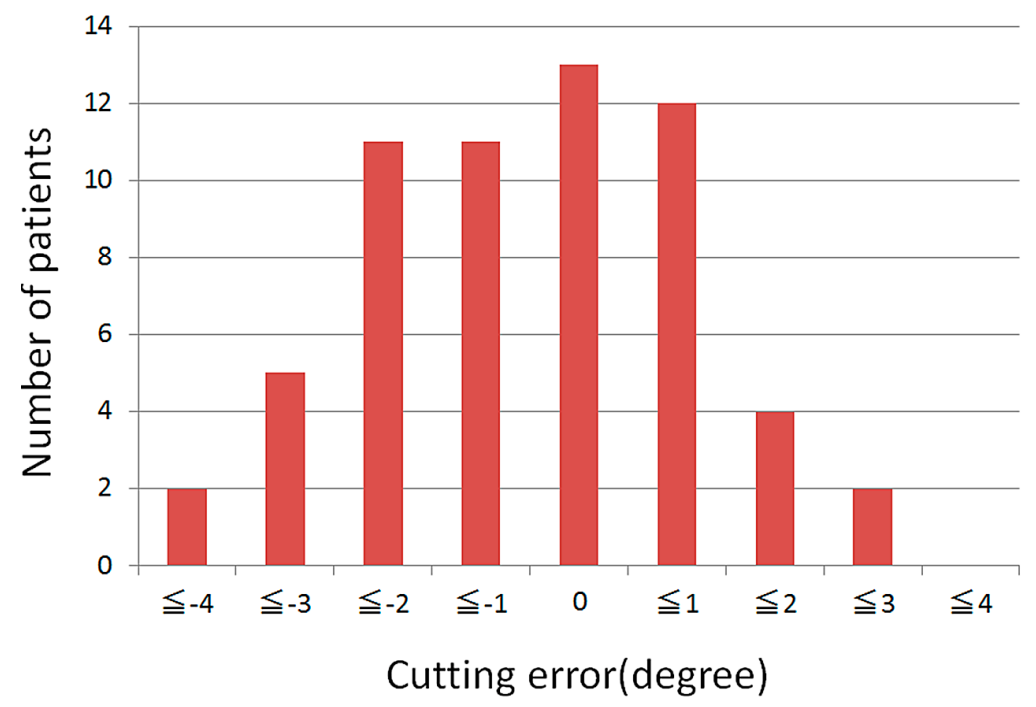

(a)

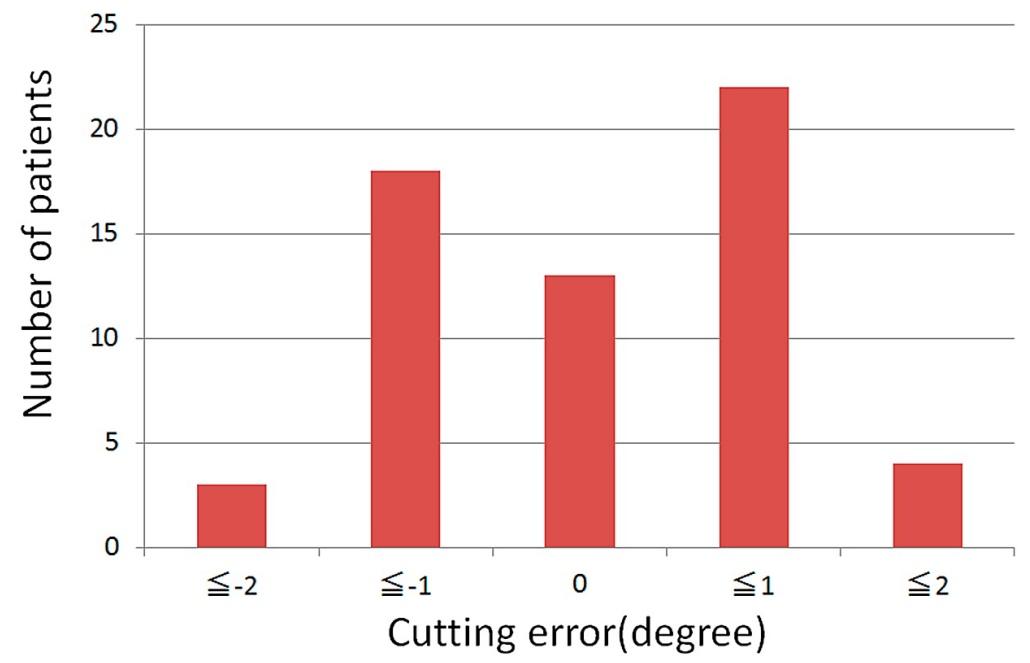

(b)

Figure 4. Proximal tibia cutting error. (a) In the sagittal plane. A positive value indicates cutting with increased posterior slope. (b) In the sagittal plane. A positive value indicates valgus position. 
Table 1. Bone cutting error relationship between the femur and the tibia in the coronal plane.

\begin{tabular}{cccccccccc}
\hline femur & valgus & valgus & valgus & varus & varus & varus & 0 & 0 & 0 \\
tibia & valgus & varus & 0 & valgus & varus & 0 & valgus & varus & 0 \\
number & 7 & 7 & 3 & 10 & 8 & 8 & 9 & 6 & 2 \\
\hline
\end{tabular}

\section{Discussion}

Accurate alignment and soft tissue balance are fundamental in a successful TKA [4] [7]. There are five main causes of malalignment: 1) inaccuracy of the preoperative plan, 2) inaccurate reproduction of the preoperative plan, 3) incorrect setting of the cutting guide, 4) inaccurate fixation of the component, and 5) incorrect cutting of the bone [8]-[11]. Accuracy of the preoperative plan can be improved by a 3D template and accuracy of the reproduction of the plan is improved by the navigation system or by PSI (patient specific instruments). Cutting guide settings also can be improved through use of a navigation system [12] [13]. Inaccurate bone cutting has been noted for a long time.

In this study, the distal femur tended to be cut in an extended and a varus position and the proximal tibia with decreased posterior slope and in a valgus position after the initial bone cutting. It tends that cutting error is larger in the sagittal plane than in the coronal plane.

Nakahara et al. reported cutting error of the distal femur in 20 knees [6]. In the sagittal plane, $13(65 \%)$ of 20 knees were cut in an extended position and in $6(30 \%)$ there was an error of more than $3^{\circ}$. Same as previous study, $37(62 \%)$ of 60 knees were cut in this study. In the sagittal plane, the bending of the bone saw was the main cause of the cutting error, causing also an incomplete cutting posteriorly [6] [8].

Insall et al. consider that an appropriately sized component is a very important prognostic factor for TKA [14]. Imamura et al. reported that an error of more than $3^{\circ}$ in the sagittal angle of the femoral distal cut can cause differences about $2 \mathrm{~mm}$ in the size of the femoral component [15]. Further Tsukeoka et al. reported that a distal femoral cutting error in $4^{\circ}$ of extension could result in component sizing $2 \mathrm{~mm}$ larger than preoperatively planned [16]. In this study, there were $9(15 \%)$ of 60 knees in which cutting error was more than $3^{\circ}$. As for the other problems associated with cutting in an extended position, a notch may be formed in the femoral anterior cortex or it can lead to overhang in association with a larger femoral component size. Tsukeoka et al. reported that a progressive increase in the degree of extension of the distal femoral cutting error in TKA resulted in a $2 \mathrm{~mm}$ increase in the flexion-extension gap difference for every $2^{\circ}$ of additional extension error [17]. Daluga et al. note that an increase in the degree of extension of the distal femoral cutting error can affect postoperative range of motion and lead to postoperative pain of the anterior knee [18].

In a sagittal plane, the tibia tended to be cut with decreased posterior slope.

A bone cutting error on the tibia can then occur due to:

1) "wobbling" of the saw blade due to saw instability;

2) bending in flexion of the blade leading to deviation of the cut;

3) wandering of the blade away from the guide surfaces (we did not use guides in this study) [17].

In this study, $29(48 \%)$ of 60 knees were cut with a decreased posterior slope on the proximal tibia side. We think flexure of the bone saw was the primary cause of the error. If the proximal tibia is cut with a decreased posterior slope, the flexion gap may be tight and range of motionmay be reduced. From the above, it is clear that we should cut bone with more precision, since cutting errors in the sagittal plane lead to restricted range of motion.

In the coronal plane, $26(43 \%)$ of 60 knees were cut in a varus on the distal femur. On the other hand, in the study by Nakahara et al., 10 (43\%) of 20 knees were cut in a valgus [6]. They explained that the first distal femur cut resulted in a slightly valgus alignment, which may be due to the fact that almost all cases had a varus deformity and the medial bone was harder than the lateral bone, which deflected the saw blade. However, our results were reversed. Also in our study, 26 (43\%) of 60 knees were cut in a valgus on the proximal tibia. Compared with the femur, bone cutting was carried out in the reverse direction. If bone cutting of the deformed side is insufficient, there should be a relationship between cutting errors of the femur and tibia, but we found no such relation in our results. So it was possible that in the coronal plane, the cutting error of femur and tibia may not be decided only by the hardness of bone cortex. Other reasons for cutting error in this study could include in stability of the cutting guide or relative motions between the cutting guide and bone saw [8] [9].

Otani et al. examined cutting error with regard to the relationship between slot and bone saw thickness [9]. 
They reported that the mean maximum cutting error at a cutting depth of $5 \mathrm{~cm}$ with a $1.2 \mathrm{~mm}$ thick saw blades was 802 microns, and with a $1.4 \mathrm{~mm}$ thick saw blades it was 488 microns. In other words, cutting error could be minimized by using thicker saw blades and a thinner slot. In this study, we used a $1.27 \mathrm{~mm}$ thick saw blade (Nakahara et al:: $0.9 \mathrm{~mm}$ ) and a $1.40 \mathrm{~mm}$ thick slot (Nakahara et al.: $1.35 \mathrm{~mm}$ ). We believe these differences in the thickness of saw blade and slot led to less motion between the cutting guide and bone saw. Further, Otani et $a l$. concluded that cutting error can be minimized by fixing the cutting guide to the distal femur with a combined fixation method utilizing a central rod and peripheral pins or clamps [9]. They reported that fixation with only pins or clamps did not provide tight fixation and had deflections from 200 microns to more than $1 \mathrm{~mm}$. In this study, because we were using a navigation system, we did not use the medullary rod; instead we used only pins, to avoid the invasive insertion of the rod. The use of three pins enabled us to achieve adequate fixation of the cutting guide. We propose a new reason why the bone was not accurately cut parallel to the AP axis in the coronal plane. When cutting a bone from the lateral or medial side, the direction of cutting moves from the AP axis. Because of incomplete bone cutting on the posterior side due to flexure of the bone saw, if we cut the bone from the lateral side, the amount of the medial bone resection decreases and, for the same reason, if we cut the bone from the medial side, the amount of the lateral bone resection also decreases. As a result, cutting errors are caused in the coronal plane. In this regard, we think it depend on which hand had performed cutting bone and ways of skin incision. About this point, the further research is required. It has been reported that a cutting error in the coronal plane of more than $3^{\circ}$ worsens the TKA prognosis [3]-[5]. But there has been no knee in which the average absolute error value was more than $2^{\circ}$. Sungkhum et al. compared the use of narrow and widesawblades on the proximal tibia bone, cut in a close slot cutting block guide and verified by a computer navigation system [2]. They did not find any statistically significant difference in their cases. However, they did find statistically significant differences in sclerotic bone. The saw blade width was $18 \mathrm{~mm}$ in this study, which is narrower than those commonly used.

There are four limitations in our study. First, we operated with the same team, but with more than one surgeon. Previous studies conclude that cutting errors are independent of the surgeon's experience [8] [19]. However, if we desire a more precise evaluation, we should operate with only one surgeon. In addition, we did not consider which side was deformed, we did not look at methods of skin incision, and we did not consider which hand was used to perform the bone cutting. These factors have a strong effect on cutting errors; they should be considered in order to evaluate cutting errors more precisely.

\section{Conclusion}

In TKA, the initial error in bone cutting has a great effect on the postoperative outcome. Especially, cutting errors in the sagittal plane tend to be larger than those in the coronal plane. The distal femur tended to be cut in an extended and varus position and the proximal tibia tended to be cut with a decreased posterior slope and valgus position. However, there were significant differences only in the sagittal plane on the distal femur. Cutting errors affect postoperative outcome. We believe that flexure of the bone saw is the main cause of the error. To improve the accuracy of one cutting, we should use thicker saw blades and a thinner slot and cut bones several times.

\section{Acknowledgements}

Special thanks are extended to Mrs. Judy for the translation of the manuscript.

\section{Conflict of Interest}

The authors declare that they have no conflict of interest.

\section{References}

[1] Matsumoto, T., Tsumura, N. and Kurosaka, M. (2004) Prosthetic Alignment and Sizing in Computer-Assisted Total Knee Arthroplasty. International Orthopaedics, 28, 282. http://dx.doi.org/10.1007/s00264-004-0562-1

[2] Sungkhun, P., Larbpaiboonpong, V. and Turajane, T. (2012) Comparing Alignment of Proximaltibia First Cut between Narrow and Wide Saw Blade in CAS TKA. Journal of the Medical Association of Thailand, 95, S87-S91.

[3] Elloy, M.A., Manning, M.P. and Johnson, R. (1992) Accuracy of Intramedullary Alignment in Total Knee Replacement. Journal of Biomedical Engineering, 14, 363-370. http://dx.doi.org/10.1016/0141-5425(92)90080-5 
[4] Jeffery, R.S., Morris, R.W. and Denham, R.A. (1991) Coronal Alignment after Total Knee Replacement. Journal of Bone and Joint Surgery (British Volume), 73, 709-714.

[5] Ritter, M.A., Faris, P.M. and Keating, E.M. (1994) Postoperative Alignment of Total Knee Replacement. Its Effect on Survival. Clinical Orthopaedics, 299, 153-156.

[6] Nakahara, H., Matsuda, S. and Morooka, T. (2012) Cutting Error of the Distal Femur in Total Knee Arthroplasty by Use of a Navigation System. Journal of Arthroplasty, 27, 1119-1122. http://dx.doi.org/10.1016/j.arth.2011.09.018

[7] Tanzer, M., Smith, K. and Burnett, S. (2002) Posterior-Stabilized versus Cruciate-Retaining Total Knee Arthroplasty: Balancing the Gap. Journal of Arthroplasty, 17, 813. http://dx.doi.org/10.1054/arth.2002.34814

[8] Plaskos, C., Hodgson, A.J. and Inkpen, K. (2002) Bone Cutting Errors in Total Knee Arthroplasty. Journal of Arthroplasty, 6, 698-705. http://dx.doi.org/10.1054/arth.2002.33564

[9] Otani, T., Whiteside, L.A. and White, L.A. (1993) Cutting Errors in Preparation of Femoral Components in Total Knee Arthroplasty. Journal of Arthroplasty, 8, 503-510. http://dx.doi.org/10.1016/S0883-5403(06)80215-9

[10] Catani, F., Biasca, N. and Ensini, A. (2008) Alignment Deviation between Bone Resection and Final Implant Positioning in Computer-Navigated Total Knee Arthroplasty. Journal of Bone and Joint Surgery (American Volume), $90,765$. http://dx.doi.org/10.2106/JBJS.G.00293

[11] Reed, S.C. and Gollish, J. (1997) The Accuracy of Femoral Intramedullary Guides in Total Knee Arthroplasty. Journal of Arthroplasty, 12, 677. http://dx.doi.org/10.1016/S0883-5403(97)90141-8

[12] Daniilidis, K. and Tibesku, C.O. (2013) Frontal Plane Alignment after Total Knee Arthroplasty Using Patient-Specific Instruments. International Orthopaedics, 37, 45-50. http://dx.doi.org/10.1007/s00264-012-1732-1

[13] Hasegawa, M., Yoshida, K. and Wakabayashi, H. (2013) Cutting and Implanting Errors in Minimally Invasive Total Knee Arthroplasty Using a Navigation System. International Orthopaedics, 37, 27-30. http://dx.doi.org/10.1007/s00264-012-1688-1

[14] Insall, J.N., Dorr, L.D. and Scott, R.D. (1998) Rationale of the Knee Society Clinical Rating System. Clinical Orthopaedics and Related Research, 13, 248.

[15] Imamura, R., Matsuda, S. and Okazaki, K. (2011) Influence on Femoral Antero-Posterior Sizing of the Error in Sagittal Angle of Femoral Distal Cut in Total Knee Arthroplasty. Journal of Orthopaedic Trauma, 60, 714-717. http://dx.doi.org/10.5035/nishiseisai.60.714

[16] Tsukeoka, T. and Lee, T.H. (2012) Sagittal Flexion of the Femoral Component Affects Flexion Gap and Sizing in Total Knee Arthroplasty. Journal of Arthroplasty, 27, 1094. http://dx.doi.org/10.1016/j.arth.2011.10.015

[17] Tsukeoka, T., Tsuneizumi, Y. and Lee, T.H. (2013) The Effect of a Sagittal Cutting Error of the Distal Femur on the Flexion-Extension Gap Difference in Total Knee Arthroplasty. Journal of Arthroplasty, 28, 1099-1102. http://dx.doi.org/10.1016/j.arth.2012.12.017

[18] Daluga, D., Lombardi Jr., A.V. and Mallory, T.H. (1991) Knee Manipulation Following Total Knee Arthroplasty: Analysis of Prognostic Variables. Journal of Arthroplasty, 6, 119. http://dx.doi.org/10.1016/S0883-5403(11)80006-9

[19] Mahaluxmivala, J., Bankes, M.J. and Nicolai, P. (2001) The Effect of Surgeon Experience on Component Positioning in 673 Press Fit Condylar Posterior Cruciate-Sacrificing Total Knee Arthroplasties. Journal of Arthroplasty, 16, 635640. http://dx.doi.org/10.1054/arth.2001.23569 